\title{
THE SYMMETRIES AND CONSERVED QUANTITIES OF MECHANICAL MULTI-BODY SYSTEM DYNAMICS
}

\author{
ZHENG Mingliang* \\ School of Mechanical and Electrical Engineering of Wuxi Taihu University, \\ Wuxi 214064, China
}

[Received: 25 January 2019. Accepted 13 May 2019]

doi: 10.7546/JTAM.49.19.04.05

\begin{abstract}
The paper provides a powerful new tool for quantitative and qualitative analysis of complex mechanical multibody system. The symmetry theory in modern mechanics is introduced into the study of mechanical multibody dynamics. Firstly, the Euler-Lagrange equations of constrained mechanical multibody system dynamics are derived by analytical mechanical method; Secondly, the theory of group analysis is introduced, according to the invariance principle, we get the conditional equations of Noether and Lie symmetry and the corresponding form of conserved quantity about mechanical multibody system, which provides an effective way to analytic integral calculate the dynamic equations. Finally, the dynamic of a bionic robot mechanism is taken as an example for practical application. The result shows that we can get deeper mechanics rules and motion phenomena of mechanical multibody system dynamics with the help of symmetries and conserved quantities, which lay a theoretical foundation for more precise dynamic optimization design and advanced control.
\end{abstract}

KEY WORDS: Mechanical multi-body system; Euler-Lagrange equations; symmetry; conserved quantity; bionic robot.

\section{INTRODUCTION}

With the development of modern science and technology, in practical engineering, especially in the fields of vehicles, spacecraft, mechanisms and robots, the complex mechanical system consisting of a large number of rigid bodies that are interlinked have emerged, which form an independent branch of discipline: multi-body system dynamics [1]. With the integration of computer technology, the modeling process and complex calculation can be programmed and simulated by software, which makes the multi-body system dynamics have made great progress and achievements in mechanical engineering [2-10]. It has been paid more and more attention by academicians and engineering scientists. According to the categories of coordinates, the mechanical multi-body system can be divided into [11]: Lagrange method, complete Carte-

\footnotetext{
${ }^{*}$ Corresponding author e-mail: zhmlwxcstu@ 163.com
} 
sian method and hybrid method. The former adopts the relative coordinates of each hinge, and the latter adopts the global coordinate system. According to the nature of objects, it can be divided into [12]: multi-rigid-body system dynamics, multi-flexiblebody system dynamics and multi-rigid-flexible-coupling-body dynamics. According to the form of equations, it can be divided into [13]: Roberson-Wittenburg method, Huston-Kane method, Lagrange method, Gauss minimum restraint principle method, spinor-matrix transformation method, Newton vector method. In either case, the basic tasks of mechanical multi-body system are as follows:

1. Establishing the general mathematical model of kinematics and dynamics.

2. Accurate calculation method for kinematic law and dynamic response.

The symmetry theory of dynamic system is a higher-level rule in theoretical physics, engineering mathematics, modern mechanics. The symmetry of motion equations is helpful to reveal the inherent characteristics and deep-seated laws of mechanical system [14]. The symmetries and conserved quantities of mechanical system are closely related, and the conserved quantity not only has obvious physical significance, but also is the first integral, which can reduce the order of equation. Therefore, symmetry theory is also an effective method to integral equation. The main symmetry methods are Noether symmetry [15], Lie symmetry [16]. However, at present, the research of mechanical multi-body system dynamics mainly adopts numerical algorithms or CAD/CAE softwares. There are few literatures on analytical integration theory. Zheng [17-19] first devoted himself to applying the symmetry theory of constrained mechanical system to mechanical multi-body system, and made a good exploration. It is well known that the analytical solution of differential equations can more directly reflect the quantitative relationship between parameters and the global properties of nonlinear system. Meanwhile, conserved quantity can also be used to design difference schemes to improve the accuracy of numerical algorithms. Therefore, it is of great significance to study the symmetry and conserved quantity of mechanical multi-body system dynamics.

\section{Dynamic Model of a Mechanical Multi-body System}

At present, the basic principles and methods for deriving control equations of mechanical multi-body system dynamic can be divided into three categories. The first one is Newton-Euler vector mechanics method; the second one is analytical mechanics method, such as Lagrange equation, Hamilton principle; the third one is extremum principle with minimum property based on Gauss principle. Considering that the analytical mechanics method is based on energy and constraints, which has the characteristics of universality and wide adaptability, and it is easy to program, which can 
simplify the control and synthesis of system. So, in this paper, the second kind of modeling method is adopted.

The position is defined by three Cartesian coordinates $(x, y, z)^{T}$ and the attitude by three Euler angles $(\phi, \theta, \varphi)^{T}$. So the generalized coordinates are by $q_{i}=$ $\left(x_{i}, y_{i}, z_{i}, \phi_{i}, \theta_{i}, \varphi_{i}\right)^{T}$, the generalized velocities are by $\left(v_{i}, k_{i}\right)=d q_{i} / d t$. The kinetic energy of a rigid body is

$$
T_{i}=\frac{1}{2} v_{i} m_{i} v_{i}^{T}+\frac{1}{2} k_{i} I_{i} k_{i}^{T} .
$$

Generalized forces acting on multi-body include gravity, active external force, spring force, damping force, friction, acting force, which can be calculated by calculating the corresponding virtual work, that is,

$$
Q_{j}=\sum_{i=1}^{n} F_{i} \frac{\partial v_{i}}{\partial q_{j}}+S_{i} \frac{\partial k_{i}}{\partial q_{j}}
$$

Here $F_{i}, S_{i}$ are the principal vectors and principal moments that the active forces acting on a rigid body $i$ simplify to its center of mass. The equations of system can be obtained by assembling a single rigid body with matrix technology.

For a tree-shaped multi-body system with holonomic and scleronomic constraints is shown in Fig. 1. The generalized coordinates are independent when the generalized coordinates are hinge coordinates and modal coordinates of deformed bodies. The dynamic equation can be obtained by using the second kind of Lagrange equations

$$
\frac{d}{d t}\left[\frac{\partial T}{\partial \dot{\mathbf{q}}}\right]-\frac{\partial T}{\partial \mathbf{q}}=\mathbf{Q} .
$$

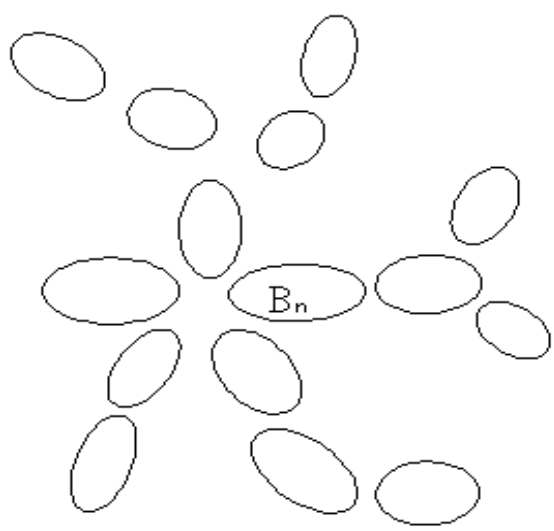

Fig. 1. Topological configuration of tree-diagram shaped multi-body system. 


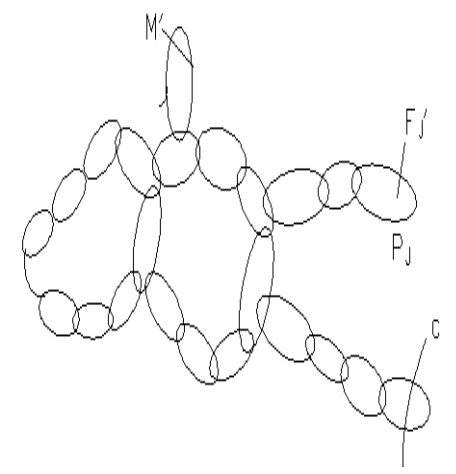

Fig. 2. Topological configuration of non-tree-shaped multi-body system.

For a complex multi-body system, the motion of components is coupled with each other. The topological structure of the non-tree-shaped multi-body system is shown in Fig. 2. Hinge coordinates are non-independent. The all Cartesian coordinates are used as the generalized coordinates, according to the topological structure and the type of hinge, the constraint equations $\Phi(\boldsymbol{q})=0, \Phi \in \boldsymbol{R}^{m}$ ( $m$ is the number of constraint equations) are established, then the dynamic equation can be obtained by using the first kind of Lagrange equations:

$$
\begin{aligned}
& \frac{d}{d t}\left[\frac{\partial T}{\partial \dot{\boldsymbol{q}}}\right]-\frac{\partial T}{\partial \boldsymbol{q}}+\Phi_{q}^{T} \boldsymbol{\lambda}=\boldsymbol{Q} \\
& \Phi(\boldsymbol{q})=0
\end{aligned}
$$

Here $\lambda$ is Lagrange constraint multipliers.

\section{Noether Symmetries ANd CONSERVEd Quantities}

The generalized force in the dynamic equation of a mechanical multi-body system is further decomposed, in which there is a potential function $V(t, \boldsymbol{q})$ can make $Q^{\prime}{ }_{s}=$ $-\partial V / \partial q_{s}$, therefore, there exists a Hamilton action form of the system

$$
S(\gamma)=\int_{t_{1}}^{t_{2}} L(t, \boldsymbol{q}, \dot{\boldsymbol{q}}) d t=\int_{t_{1}}^{t_{2}} T-V d t .
$$

Taking infinitesimal transformation of time and coordinates

$$
t^{*}=t+\Delta t=t+\varepsilon \xi_{0}(t, \boldsymbol{q}, \dot{\boldsymbol{q}}), q_{s}^{*}=q_{s}(t)+\Delta q_{s}=q_{s}(t)+\varepsilon \xi_{s}(t, \boldsymbol{q}, \dot{\boldsymbol{q}}) .
$$

Here, $\xi_{0}, \xi_{s}$ are infinitesimal generating functions. 
Generalized quasi-Noether symmetry means that the total variation of (5) satisfies under (6):

$$
\Delta S=-\int_{t_{1}}^{t_{2}}\left\{\frac{d}{d t}(\Delta G)+\sum_{i=1}^{n} Q_{s}^{\prime \prime} \delta q_{s}\right\} d t .
$$

Here $\Delta G=\varepsilon G_{N}(t, \boldsymbol{q}, \dot{\boldsymbol{q}}), G_{N}$ is a gauge function, $Q^{\prime \prime}{ }_{s}$ is non-potential force.

According to the relation between total variation and isochronal variation

$$
\delta q_{s}=\Delta q_{s}-\dot{q}_{s} \Delta t=\varepsilon\left(\xi_{s}-\dot{q}_{s} \xi_{0}\right),
$$

equation (7) can be expanded

$$
\text { (8) } \begin{aligned}
\frac{\partial L}{\partial t} \xi_{0}+\sum_{s=1}^{n} \frac{\partial L}{\partial q_{s}} \xi_{s} & +\sum_{s=1}^{n} \frac{\partial L}{\partial \dot{q}_{s}} \dot{\xi}_{s} \\
& +\left(L-\sum_{s=1}^{n} \frac{\partial L}{\partial \dot{q}_{s}} \dot{\xi}_{s}\right) \dot{\xi}_{0}+\sum_{s=1}^{n} Q_{s}^{\prime \prime}\left(\xi_{s}-\dot{q}_{s} \xi_{0}\right)+\dot{G}_{N}=0 .
\end{aligned}
$$

Equation (8) is the deterministic equation of generators $\xi_{0}, \xi_{s}$ and satisfies the Noether symmetry.

Noether symmetry of mechanical multi-body system with external constraints must be another condition, that is, the constraints must remain unchanged under (6). Therefore, there exists an additional restriction equation

$$
\frac{\partial \Phi(\boldsymbol{q})}{\partial q_{s}} \xi_{s}=0
$$

Therefore, the deterministic equation of Noether symmetry are (8) for tree-shaped mechanical multi-body system, but the deterministic equations of Noether symmetry are (8) and (9) for non-tree-shaped multi-body system.

The symmetry of continuous transformation corresponds to a conservation law. A conserved quantity corresponds to the first integral of system, and then the differential equation can be reduced. The conserved quantity is a deeper dynamic law, which plays an important role in the integrability, linearization, motion constants and stability of dynamic equation. Noether symmetry can directly lead to a class of Noether-type conserved quantities, and combining with the initial conditions, it is easy to solve the exact response of original vibration system. The form of Noethertype conserved quantity is

$$
I_{N}=L \xi_{0}+\sum_{s=1}^{n} \frac{\partial L}{\partial \dot{q}_{s}}\left(\xi_{s}-\dot{q}_{s} \xi_{0}\right)+G_{N}=\text { const. }
$$

Integrating the left of (10), we have $d I_{N} / d t=0$. 


\section{Lie Symmetries AND CONSERVED QuANTITIES}

Unlike Noether's theory, Lie symmetry is a directly study the invariance of differential equations under a infinitesimal transformation. All generalized accelerations can be obtained by expanding the dynamic equations of tree-shaped and non-tree-shaped multi-body systems under the condition of non-singularity. So,

$$
\ddot{q}_{s}=\alpha_{s}(t, \boldsymbol{q}, \operatorname{dot} \boldsymbol{q}) \quad(s=1,2, \ldots, n) .
$$

Lie symmetry of mechanical multi-body system means that Eq. (11) remains unchanged under the infinitesimal transformation (6). That is,

$$
\ddot{q}_{s}^{*}=\alpha_{s}\left(t^{*}, \boldsymbol{q}^{*}, \dot{\boldsymbol{q}}^{*}\right) .
$$

Equation (12) can also be expressed as the deterministic equation of Lie symmetry

$$
\ddot{\xi}_{s}-\dot{q}_{s} \ddot{\xi}_{0}-2 \alpha_{s} \dot{\xi}_{0}=\frac{\partial \alpha_{s}}{\partial t}+\sum_{k=1}^{n} \frac{\partial \alpha_{s}}{\partial q_{k}} \xi_{k}+\sum_{k=1}^{n} \frac{\partial \alpha_{s}}{\partial \dot{q}_{k}}\left(\dot{\xi}_{k}-\dot{q}_{k} \dot{\xi}_{0}\right) .
$$

Equation (13) is the deterministic equation of Lie symmetry for tree-shaped mechanical multi-body system.

But the Lie symmetry of mechanical multi-body system with external constraints must also satisfy the additional restrictive equation (9), so the deterministic equation of Lie symmetry are (13) and (9) for non-tree-shaped multi-body system..

Unlike Noether symmetry, Lie symmetry does not directly lead to conserved quantity in general. The condition and form of conserved quantity caused by Lie symmetry are given below.

If the generators satisfy the deterministic equation of Lie symmetry and there is a gauge function $G_{N}=G_{N}(t, \boldsymbol{q}, \dot{\boldsymbol{q}})$ that satisfies the structural equation

$$
L \dot{\xi}_{0}+\xi_{0} \frac{\partial L}{\partial t}+\sum_{k=1}^{n} \xi_{k} \frac{\partial L}{\partial q_{k}}+\sum_{k=1}^{n}\left(\dot{\xi}_{s}-\dot{q}_{s} \dot{\xi}_{0}\right) \frac{\partial L}{\partial \dot{q}_{s}}+\sum_{i=1}^{n} Q_{s}^{\prime \prime}\left(\xi_{s}-\dot{q}_{s} \xi_{0}\right)+\dot{G}_{N}=0
$$

Then Lie symmetry can lead to Noether-type conserved quantity

$$
I_{N}=L \xi_{0}+\sum_{i=1}^{n} \frac{\partial L}{\partial \dot{q}_{s}}\left(\xi_{s}-\dot{q}_{s} \xi_{0}\right)+G_{N}=\text { const. }
$$

Integrating the left side of (15), we obtain $d I_{N} / d t=0$.

Lie symmetry can also construct a new type of conserved quantities under certain conditions, which does not depend on the Lagrange function. That is, if the generators satisfy the deterministic equation and a gauge function $\mu=\mu(t, \boldsymbol{q}, \dot{\boldsymbol{q}})$ satisfies 
the structural equation

$$
\sum_{s=1}^{n} \frac{\partial \alpha_{s}}{\partial \dot{q}_{s}}+\frac{d}{d t} \ln \mu=0 .
$$

Then Lie symmetry can also lead to Hojman-type conserved quantity

$$
\begin{aligned}
I=\frac{\partial \xi_{0}}{\partial t}+\sum_{s=1}^{n} \frac{\partial \xi_{s}}{\partial q_{s}} & +\sum_{s=1}^{n} \frac{\partial\left(\dot{\xi}_{s}-\dot{q}_{s} \dot{\xi}_{0}\right)}{\partial \dot{q}_{s}}+\xi_{0} \frac{\partial \ln \mu}{\partial t} \\
& +\sum_{s=1}^{n} \xi_{s} \frac{\partial \ln \mu}{\partial q_{s}}+\sum_{s=1}^{n}\left(\dot{\xi}_{s}-\dot{q}_{s} \dot{\xi}_{0}\right) \frac{\partial \ln \mu}{\partial \dot{q}_{s}}-\xi_{0}=\text { const. }
\end{aligned}
$$

Integrating the left of (17), we have $d I / d t=0$.

\section{EXAMPLE ILLUSTRATION}

The sideways motion model of a bionic robot can be regarded as a multi-body system consisting of five connecting rods and four rotating joints mechanisms [20]. The geometry is shown in Fig. 3.

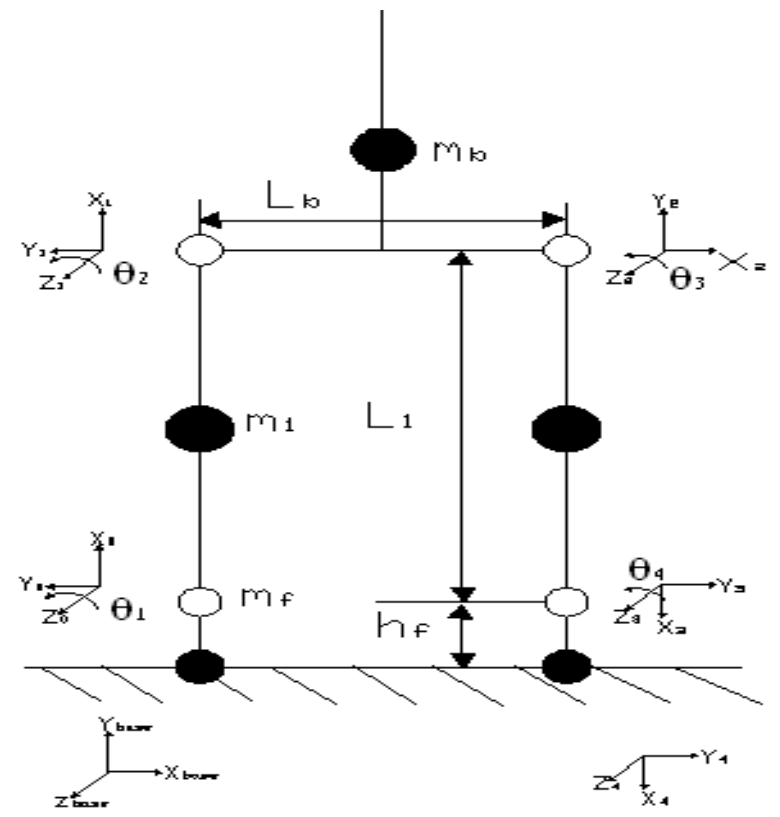

Fig. 3. The sideways geometric model of bionic robot. 
In order to operate effectively, the planning upper body posture of robot remains unchanged during walking, so $\theta_{2}=-\theta_{3}, \theta_{1}=-\theta_{4}$, the torques acting on joints 3 and 4 are $\tau_{3}, \tau_{4}$, the independent generalized coordinates are $\theta_{3}, \theta_{4}$, the Lagrange function is

$$
\begin{aligned}
L=\frac{1}{8}\{ & -8 g h_{f}\left(2 m_{1}+m_{b}+m_{f}\right) \\
& +L_{b}\left[4\left(2 g S_{34}+\dot{\theta}_{3}^{2} L_{b}+2 \dot{\theta}_{3} \dot{\theta}_{4} L_{b}+\dot{\theta}_{4}^{2} L_{b}\right) m_{1}\right. \\
& +\left(4 g S_{34}+\dot{\theta}_{3}^{2} L_{b}+2 \dot{\theta}_{3} \dot{\theta}_{4} L_{b}+\dot{\theta}_{4}^{2} L_{b}\right) m_{b} \\
& \left.+4\left(2 g S_{34}+\dot{\theta}_{3}^{2} L_{b}+2 \dot{\theta}_{3} \dot{\theta}_{4} L_{b}+\dot{\theta}_{4}^{2} L_{b}\right) m_{f}\right] \\
& +4 L_{1}\left[\left(4 g S_{4}+3 C_{3} \dot{\theta}_{3} \dot{\theta}_{4} L_{b}+3 C_{3} \dot{\theta}_{4}^{2} L_{b}\right) m_{1}\right. \\
& +\left(2 g S_{4}+C_{3} \dot{\theta}_{3} \dot{\theta}_{4} L_{b}+C_{3} \dot{\theta}_{4}^{2} L_{b}\right) m_{b} \\
& \left.+4\left(g S_{4}+C_{3} \dot{\theta}_{3} \dot{\theta}_{4} L_{b}+C_{3} \dot{\theta}_{4}^{2} L_{b}\right) m_{f}\right] \\
& \left.+2 \dot{\theta}_{4}^{2} L_{1}^{2}\left[5 m_{1}+2\left(m_{b}+m_{f}\right)\right]\right\} .
\end{aligned}
$$

Here $C_{3}$ and $C_{4}$ are $\cos \left(\theta_{3}\right), \cos \left(\theta_{4}\right) ; C_{3+4}$ and $C_{3-4}$ are $\cos \left(\theta_{3}+\theta_{4}\right), \cos \left(\theta_{3}-\theta_{4}\right)$; $S_{3}$ and $S_{4}$ are $\sin \left(\theta_{3}\right), \sin \left(\theta_{4}\right) ; S_{3+4}$ and $S_{3-4}$ are $\sin \left(\theta_{3}+\theta_{4}\right), \sin \left(\theta_{3}-\theta_{4}\right)$.

The multi-body system is a tree model, and the structural parameters of robot are substituted into the Euler-Lagrange equation to obtain

$$
\begin{aligned}
\tau_{3}= & \frac{1}{4}\left\{4 h_{j}\left(g-C_{34} \ddot{\theta}_{3} L_{b}-C_{34} \ddot{\theta}_{3} L_{b}\right) m_{f}-\ddot{\theta}_{4} L_{1}^{2}\left(3 m_{1}+8 m_{f}\right)\right. \\
& +2 L_{1}\left[\left(g C_{4}-C_{3} \ddot{\theta}_{3} L_{b}-C_{3} \ddot{\theta}_{4} L_{b}\right) m_{1}\right. \\
& \left.\left.+2\left(g C_{4}-C_{3} \ddot{\theta}_{3} L_{b}-\ddot{\theta}_{4}\left(2 C_{4} h_{f}+C_{3} L_{b}\right)\right) m_{f}\right]\right\}, \\
\tau_{4}= & h_{f}\left[g-C_{34} \ddot{\theta}_{3} L_{b}-\ddot{\theta}_{4}\left(2 C_{4} L_{1}+C_{34} L_{b}\right)\right] m_{f} .
\end{aligned}
$$

Now we study the Lie symmetry and conserved quantity. The expanded form of deterministic equation of Lie symmetry are

$$
\begin{aligned}
& -\left[2 \dot { \xi } _ { 0 } \left[4\left(-\tau_{3}+\tau_{4}\right) C_{34} h_{f} L_{b} m_{f}\right.\right. \\
& \quad+L_{1}^{2}\left(m_{1}\left[3 \tau_{4}+g\left(-1+2 C_{44}\right) h_{f} m_{f}\right]+8 m_{f}\left(\tau_{4}-g S_{4}^{2} h_{f} m_{f}\right)\right) \\
& \left.\left.\quad+L_{1}\left[8\left(-\tau_{3}+\tau_{4}\right) C_{4} h_{f} m_{f}+L_{b}\left(m_{1}+2 m_{f}\right)\left(2 \tau_{4} C_{3}-2 g S_{4} S_{34} h_{f} m_{f}\right)\right]\right]\right] \\
& \quad \times\left[h_{f} L_{1}^{2} L_{b} m_{f}\left[\left(C_{3} C_{4}+3 S_{3} S_{4}\right) m_{1}+8 S_{3} S_{4} m_{f}\right]\right]^{-1}-\dot{\theta}_{3} \ddot{\xi}_{0}+\ddot{\xi}_{1}=
\end{aligned}
$$




$$
\begin{aligned}
=- & \left\{2 S_{4} L_{b}\left(m_{1}+2 m_{f}\right)+L_{1}\left[\left(-C_{4} S_{3}+3 C_{3} S_{4}\right) m_{1}+8 C_{3} S_{4} m_{f}\right]\right\} \\
& \times\left\{8\left(-\tau_{3}+\tau_{4}\right) C_{4} h_{f} m_{f}+L_{1}\left\{m_{1}\left[3 \tau_{4}+g\left(-1+2 C_{44}\right) h_{f} m_{f}\right]\right.\right. \\
& \left.\left.+8 m_{f}\left(\tau_{4}-g S_{4}^{2} h_{f} m_{f}\right)\right\}\right\} \xi_{1}+\left\{\left[2 h _ { f } m _ { f } \left[-\left(C_{3} C_{4}+3 S_{3} S_{4}\right) m_{1}\right.\right.\right. \\
& \left.-8 S_{3} S_{4} m_{f}\right]\left\{2\left(-\tau_{3}+\tau_{4}\right) S_{34} L_{b}+2 g S_{44} L_{1}^{2}\left(m_{1}+2 m_{f}\right)\right. \\
& \left.+L_{1}\left[4\left(-\tau_{3}+\tau_{4}\right) S_{4}+g S_{34} L_{b}\left(m_{1}+2 m_{f}\right)\right]\right\}-\left[\left(2 S_{3-4}+S_{34}\right) m_{1}\right. \\
& \left.+8 C_{4} S_{3} m_{f}\right]\left\{4\left(-\tau_{3}+\tau_{4}\right) C_{34} h_{f} m_{f} L_{b}+L_{1}^{2}\left\{m _ { 1 } \left[3 \tau_{4}+g(-1\right.\right.\right. \\
& \left.\left.\left.+2 C_{44}\right) h_{f} m_{f}\right]+8 m_{f}\left(\tau_{4}-g S_{4}^{2} h_{f} m_{f}\right)\right\}+L_{1}\left[8\left(-\tau_{3}+\tau_{4}\right) C_{4} h_{f} m_{f}\right. \\
& \left.\left.\left.\left.+L_{b}\left(m_{1}+2 m_{f}\right)\left(2 \tau_{4} C_{3}-2 g S_{4} S_{34} h_{f} m_{f}\right)\right]\right\}\right\} \xi_{2}\right] \\
& \times\left[h_{f} L_{1}^{2} L_{b} m_{f}\left[\left(C_{3} C_{4}+3 S_{3} S_{4}\right) m_{1}+8 S_{3} S_{4} m_{f}\right]^{2}\right]^{-1} \cdot \\
-\left[2 \dot{\xi}_{0}\right. & {\left.\left[4\left(\tau_{3}-\tau_{4}\right) C_{34} h_{f} m_{f}+L_{1}\left(m_{1}+2 m_{f}\right)\left(-\tau_{4} C_{3}+g S_{4} S_{34} h_{f} m_{f}\right)\right]\right] } \\
\times & {\left[h_{f} L_{1}^{2} m_{f}\left[\left(C_{3} C_{4}+3 S_{3} S_{4}\right) m_{1}+8 S_{3} S_{4} m_{f}\right]\right]-\dot{\theta}_{4} \ddot{\xi}_{0}+\ddot{\xi}_{2}=} \\
=[\{2 & S_{4}\left(m_{1}+2 m_{f}\right)\left\{8\left(-\tau_{3}+\tau_{4}\right) C_{4} h_{f} m_{f}+L_{1}\left\{m _ { 1 } \left[3 \tau_{4}+g(-1\right.\right.\right. \\
& \left.\left.\left.\left.+2 C_{44}\right) h_{f} m_{f}\right]+8 m_{f}\left(\tau_{4}-g S_{4}^{2} h_{f} m_{f}\right)\right\}\right\} \xi_{1}+\left\{2 h _ { f } m _ { f } \left[-\left(C_{3} C_{4}\right.\right.\right. \\
& \left.\left.+3 S_{3} S_{4}\right) m_{1}+8 S_{3} S_{4} m_{f}\right]\left[2\left(-\tau_{3}+\tau_{4}\right) S_{34}+g S_{44} L_{1}^{2}\left(m_{1}+2 m_{f}\right)\right] \\
& -\left[\left(2 S_{3-4}+S_{34}\right) m_{1}+8 C_{4} S_{3} m_{f}\right]\left[4\left(\tau_{3}-\tau_{4}\right) C_{34} h_{f} m_{f}\right. \\
& \left.\left.\left.+2 L_{1}\left(m_{1}+2 m_{f}\right)\left(-\tau_{4} C_{3}+g S_{4} S_{34} h_{f} m_{f}\right)\right]\right\} \xi_{2}\right] \\
\times & {\left[h_{f} L_{1}^{2} m_{f}\left[\left(C_{3} C_{4}+3 S_{3} S_{4}\right) m_{1}+8 S_{3} S_{4} m_{f}\right]^{2}\right] } \\
& +1
\end{aligned}
$$

Equation (20) is a coupled complex nonlinear differential equations, we have the solution

$$
\xi_{0}=c, \quad \xi_{1}=0, \quad \xi_{2}=0 .
$$

Then the structural equation is

$$
-c \tau_{3} \dot{\theta}_{3}-c \tau_{4} \dot{\theta}_{4}+\dot{G}=0 .
$$

Then the gauge function is

$$
G=c \int\left(\tau_{3} d \theta_{3}+\tau_{4} d \theta_{4}\right) .
$$


Then the corresponding conserved quantity is

$$
\begin{aligned}
I=\frac{1}{8}[ & -8 g h_{f}\left(2 m_{1}+m_{b}+m_{f}\right) \\
& -L_{b}\left[4\left(-2 g S_{34}+\dot{\theta}_{3}^{2} L_{b}+2 \dot{\theta}_{3} \dot{\theta}_{4} L_{b}+\dot{\theta}_{4}^{2} L_{b}\right) m_{1}\right. \\
& +\left(-4 g S_{34}+\dot{\theta}_{3}^{2} L_{b}+2 \dot{\theta}_{3} \dot{\theta}_{4} L_{b}+\dot{\theta}_{4}^{2} L_{b}\right) m_{b} \\
& \left.+4\left(-2 g S_{34}+\dot{\theta}_{3}^{2} L_{b}+2 \dot{\theta}_{3} \dot{\theta}_{4} L_{b}+\dot{\theta}_{4}^{2} L_{b}\right) m_{f}\right] \\
& +4 L_{1}\left[\left(4 g S_{4}-3 C_{3} \dot{\theta}_{3} \dot{\theta}_{4} L_{b}-3 C_{3} \dot{\theta}_{4}^{2} L_{b}\right) m_{1}\right. \\
& +\left(2 g S_{4}-C_{3} \dot{\theta}_{3} \dot{\theta}_{4} L_{b}-C_{3} \dot{\theta}_{4}^{2} L_{b}\right) m_{b} \\
& \left.+4\left(g S_{4}-C_{3} \dot{\theta}_{3} \dot{\theta}_{4} L_{b}-C_{3} \dot{\theta}_{4}^{2} L_{b}\right) m_{f}\right] \\
& \left.-2 \dot{\theta}_{4}^{2} L_{1}^{2}\left[5 m_{1}+2\left(m_{b}+4 m_{f}\right)\right]\right]+\int\left(\tau_{3} d \theta_{3}+\tau_{4} d \theta_{4}\right)=\text { const. }
\end{aligned}
$$

Up to now, a new conserved quantity different from energy conservation law, momentum conservation law and angular momentum conservation law are obtained. By substituting the specific value of variables $\theta_{3}, \theta_{4}, \tau_{3}, \tau_{4}$, a more specific conserved quantity can be obtained. For the dynamics of robot multi-body system, the conservation law is successfully obtained by using Lie symmetry theory, which is seldom involved in the relevant literature at present. These contents in this paper will enhance the understanding of inherent physical nature of sideways motion of bionic robot and promote the research of bionic robot dynamics.

\section{CONCLusions}

Symmetry theory is rich and has broad application prospects in mathematical science and engineering technology. It is necessary to study the integral theory of mechanical multi-body system dynamics although considering the premise of computer technology. In this paper, the research contents of mechanical multi-body systems are summarized, which includes the mathematical modeling of dynamic equation, Noether symmetry and conserved quantity, Lie symmetry and conserved quantity. The conserved quantity of bionic robot system is obtained by using Lie symmetry method, which unlike any existing method. The symmetric method is easy to standardize, and with the introduction of symbolic software technology, the solution of procedure will be very efficient. We can not only get the first integral of dynamic response of system, but also get an effective mean to verify the numerical calculation. Furthermore, the method can be extended to the study of dynamics of mechanical multi-body system with complex boundary constraints (non-ideal). 


\section{REFERENCES}

[1] W.U. Hongtao, Xiong Youlun (2000) The Problem of Multi-body Systems Dynamics in Mechanical Engineering. China Mechanical Engineering 11(8) 608-610 (in Chinese).

[2] W. SCHIEHLen (1997) Multibody System Dynamics: Roots and Perspectives. Multibody System Dynamics 1(2) 149-188.

[3] T.M. Wesfy, A.K. Noor (2003) Computational Strategies for Flexible Multibody Systems. Applied Mechanics Reviews 56(6) 553-613.

[4] Y.A. HuAng, Z.C. Deng, Y.L. XIONG (2007) Dynamic Analysis of a Rotating RigidFlexible Coupled Smart Structure with Large Deformations. Applied Mathematics and Mechanics 28(10) 1349-1360.

[5] P. Zhengkuang, Z. Weijia, H. JiaZheng, et Al. (1996) On Numerical Algorithms for Differential/Algebraic Equations of Motion of Multibody Systems. Advances In Mechanics 26(1) 28-40 (in Chinese).

[6] W. QI (1996) Implicit Numerical Algorithm for Multibody Dynamics with Open Loop Systems. Acta Mechanica Sinica 1(6) 8-12 (in Chinese).

[7] X. Xiaoming, Z. WAnXIE (2014) Symplectic Integration for Multibody Dynamics Based on Quaternion Parameters. Applied Mathematics and Mechanics 35(10) 10711080 (in Chinese).

[8] L. Caishan, L. Youwu, W. Jianming (1998) Dynamic Modeling and Variable Structure Control of a Flexible Beam. Journal of Vibration and Shock 17(1) 24-29 (in Chinese).

[9] W. OSKaR, W. Simon (2002) Simulation of Deployment of a Flexible Solar Array. Multibody System Dynamics 7 101-125.

[10] P. CARLBOM (2001) Combining MBS with FEM for Rail Vehicle Dynamics Analysis. Multibody System Dynamics 6(3) 291-300.

[11] H. JIAZHEN (1992) "Multibody System Dynamics Theory, Calculating and Application”. Shanghai Jiaotong University Press. (in Chinese).

[12] H. Wenhu (1996) "Flexible Multibody System Dynamics". Science Press. (in Chinese).

[13] H. Xiaohua, W. Xingcheng (2007) Lie Group Representation of Robot Dynamics and its Applications. China Mechanical Engineering 18(2) 201-205 (in Chinese).

[14] M. FengXiang (1999) "Applications of Lie Groups and Lie Algebras to Constrained Mechanical Systems". Science Press (in Chinese).

[15] E. Noether (1918) Invariante Variationsprobleme. Nachr. König. Gesell. Wissen. Göttingen, Mathematical Physics KI, II 235-257.

[16] M. LutZKY (1979) Dynamical Symmetries and Conserved Quantities. Journal of Physics A: Mathematical and General 12(7) 973-981.

[17] Z. Mingliang (2018) The Noether Theorem for Nonlinear Optimal Control Problems of Mechanical Multibody System Dynamics. Applied Mathematics and Mechanics 39(7) 776-784 (in Chinese). 
[18] Z. Mingliang, F. Xian, L. Wenxia, C. Yalin (2018) Study on Symmetries and Conserved Quantities of Mechanical Multibody System Collision Dynamics. Applied Mathematics and Mechanics 39(11) 1292-1299 (in Chinese).

[19] Z. Mingliang (2018) Lie Group Analysis Method for Structural Dynamic Equation of Mechanical System. Light Industry Machinery 36(1) 1-4 (in Chinese).

[20] K.E. XIAngXIN (2005) "Bipedal Dynamic Walking for a Humanoid Robot". Shanghai University (in Chinese). 